\title{
Bone physiology and bone healing
}

\author{
December 2002
}

\author{
Michael A. Morone, M.D., Ph.D. \\ Department of Neurosciences, Deaconess Billings Clinic, Billings Montana
}

In the not-too-distant past, spine surgeons performed spinal arthrodesis procedures and bone union would occur. This wish, however, was often not realized, and a nonunion (pseudarthrosis) developed in 10 to $45 \%$ of patients. Although the introduction of adjunctive spinal instrumentation reduced the nonunion rate, it remained significant, ranging from 5 to $15 \%$. At present, instrumentation-assisted biomechanical stabilization is probably at its most effective in preventing nonunion, and it is unlikely that additional technical advances in instrumentation will further reduce the nonunion rate. Realizing this, in the early 1990s investigators such as Scott D. Boden and colleagues approached the problem in a different manner by examining why spinal nonunion occurs. They examined the biological phenomenon of spinal bone healing after arthrodesis, hoping to determine biological strategies that reduce or eliminate the development of a pseudarthrosis. These investigators realized that considerable spinal healing information had been extrapolated from studies involving long bone fracture healing. Although similarities are present between long bone healing and vertebral healing, significant differences exist, including the more difficult healing environment in the spine (especially with an often poor blood supply), more reliance on graft material, and, especially in cases of posterior spinal arthrodesis, less of a biomechanical load applied to the fused segment.

In this issue of Neurosurgcial Focus, we continue the discussion started in an earlier issue edited by Dr. Regis W. Haid on bone fusion (Neurosurgical Focus, April 2001, Volume 10, Issue 4). This issue begins with two articles from Wayne State University. In the first Pilitsis, et al., review the biological basics of bone healing and spinal fusion. In the second Rengachary discusses the basic concepts of the bone morphogenetic proteins (BMPs). Walker and Wright then provide a timely and comprehensive review of the experimental and clinical evidence of the effectiveness of BMPs in promoting spinal fusion, with particular attention paid to the most commonly studied BMP, BMP-2. A review of the literature by Govender, et al., concerning the use of another BMP, osteogenic protein (OP)-1 (BMP-7), in spinal surgery follows, including a prospectively studied series of patients at high risk of developing a postoperative pseudarthrosis. An often overlooked or unrealized strategy to enhance fusion is the use of electrical stimulation (ES). Its infrequent application may be due to a lack of knowledge by many spine surgeons, especially neurosurgeons, of its positive effects on inducing bone growth. In a paper coauthored with one of my former colleagues in Indianapolis, Henry Feuer, we discuss the biological effects of ES on spinal fusion. Additionally experimental and clinical evidence is comprehensively reviewed, and the different types of clinically available ES are discussed. To combat this pseudarthrosis problem, we hope to continue to promote electronic dialogue on the use of ES-induced bone healing and the dissemination of strategies as they occur in the future. This ongoing discussion will be possible with Neurosurgical Focus, in which future manuscripts can still be submitted, even after the online publication of this issue. We will continue to accept manuscripts and if accepted they will be linked back to this issue.

As with each issue of Neurosurgical Focus, assorted articles are included. In this edition, Malone, et al., review the chiropractic-related complications of cervical spinal manipulation in their region of the country.

I would like to acknowledge the significant effort provided by several individuals in compiling this issue of Neurosurgical Focus. With much appreciation I would like to thank Susan M. Lanterman of Neurosurgical Focus for her guidance and Jenny J. Ragsdale for her tireless efforts. Much gratitude is also expressed for the reviewers of the submitted manuscripts and their attention to detail. Finally, I would like to thank the authors who took the time and effort to submit articles for this issue. 